\title{
A Reassessment of Weaning Parameters in Patients With Spontaneous Intracerebral Hemorrhage
}

Paras Savla ${ }^{1}$, Harjyot Toor ${ }^{1}$, Stacey Podkovik ${ }^{1}$, Joseph Mak ${ }^{2}$, Sarala Kal ${ }^{3}$, Chantal Soliman ${ }^{3}$, Andrew Ku ${ }^{4}$, Gohar Majeed ${ }^{1}$, Dan E. Miulli ${ }^{5}$

1. Neurosurgery, Riverside University Health System Medical Center, Moreno Valley, USA 2. Internal Medicine, University of California Riverside School of Medicine, Riverside, USA 3. Neurosurgery, St. George's University School of Medicine, St. George, GRD 4. Neurosurgery, California University of Science and Medicine, Colton, USA 5. Neurosurgery, Arrowhead Regional Medical Center, Colton, USA

Corresponding author: Paras Savla, paras.a.savla@gmail.com

\section{Abstract}

\section{Background and purpose}

Patients with spontaneous intracerebral haemorrhage have significant morbidity and mortality. One aspect of their care is the need for mechanical ventilation. Extubating a patient safely and efficiently is important in advancing their care; however, traditional extubation criteria using the rapid shallow breathing index and negative inspiratory force do not predict success in these patients as well as they do in other intubated patients. This study aimed to evaluate these criteria in patients with spontaneous intracerebral haemorrhage to improve the extubation success rate.

\section{Methods}

We conducted a retrospective chart review of patients with spontaneous intracerebral haemorrhage (SICH) who underwent spontaneous breathing trials from 2018 to 2020. Twenty-nine patients met the inclusion criteria, and of these 29, 20 had a trial of extubation. Rapid shallow breathing index (RSBI), negative inspiratory force (NIF), and cuff leak were recorded to analyze breathing parameters at the time of extubation. Patients who required reintubation were noted.

\section{Results}

All trials of extubation required a cuff leak. Using RSBI, patients with values $<105$ or $<85$, as the only other extubation criteria, were associated with a $70.6 \%$ and $71.4 \%$ success rate, respectively. With RSBI $<105$ and $\mathrm{NIF}<-25 \mathrm{~cm}$ water, the success rate was $88.9 \%$. Any patient with a cuff leak that had a NIF $<-30$ had a success rate of $100 \%$, regardless of RSBI.

Received 11/09/2020

Review began $11 / 25 / 2020$ Review ended 12/22/2020 Published 01/06/2021

๑) Copyright 2021 Savla et al. This is an open access article distributed under the terms of the Creative Commons Attribution License CC-BY 4.0., which permits unrestricted use, distribution, and reproduction in any medium, provided the original author and source are credited.

\section{Conclusion}

The RSBI was not a reliable isolated measure to predict $100 \%$ extubation success. Using a NIF <-30 predicts a $100 \%$ extubation success rate if a cuff leak is present. This demonstrates that the NIF may be a more useful metric in sICH patients, as it accounts for patient participation and innate ability to draw a breath spontaneously. Future studies are warranted to evaluate further and optimize the extubation criteria in these patients.

Categories: Neurosurgery, Pulmonology

Keywords: intracerebral hemorrhage, extubation, spontaneous breathing trial, rsbi, rapid shallow breathing index, nif, ich, tracheostomy, extubation failure, negative inspiratory force

\section{Introduction}

The outcome of spontaneous intracerebral haemorrhage (sICH) patients remains grim, especially in patients requiring mechanical ventilation. Families of sICH patients will often ask physicians to predict survival, independence, and need for prolonged ventilatory support. Although several prognostic models and scores were designed to predict favourable vs unfavourable outcome or death following sICH, none were outlined to predict the need for prolonged ventilation reliably and ultimately tracheostomy $[1,2]$.

Many sICH patients with poor mental status are at risk of airway compromise and require intubation and mechanical ventilation (MV) [3]. Some will ultimately get extubated. The rest will require a tracheostomy for long-term airway support. In general, tracheostomy is usually indicated after 14-21 days of MV not only to prevent complications of prolonged intubation such as vocal cord injury, tracheomalacia, and ventilatorassociated pneumonia but also to facilitate discharge of the patient from the intensive care unit (ICU) [4]. Extubation delay is associated with increased morbidity, mortality, length of ICU and hospital stay, and 
increased hospital charges [5]. However, failed extubation and the need for reintubation also serves as a major cause of morbidity and mortality in these patients [6].

Traditionally, two parameters in addition to cuff leak used in determining extubation trials are the RSBI and NIF. The RSBI is the ratio of respiratory frequency (f) to tidal volume (VT). The NIF relies on a patient's ability to generate spontaneous breathing which occurs maximally by following commands. These values have been studied, and cutoffs have been generated to reach a satisfactory successful extubation rate [7,8]. However, these respiratory weaning parameters are not always reliable in brain injury patients, including those with sICH due to the inability to achieve maximal spontaneous inspiration $[8,9]$. Although several predisposing factors, such as the presence of obstructive lung disease, hematoma volume, poor Glasgow Coma Scale (GCS), basal ganglia haemorrhage, presence of hydrocephalus and loss of brainstem reflexes, suggest a greater likelihood of failed extubation, intensivists continue to use cuff leak, RSBI primarily, and NIF to make decisions regarding extubation trials $[10,11]$.

Our study seeks to determine the optimal cutoffs of RSBI and NIF, in addition to cuff leak, for successful extubation in patients with sICH. We evaluate patients with sICH and their breathing parameters with reintubation and tracheostomy rates.

\section{Materials And Methods}

We conducted the study at a single institution using a retrospective chart review. Patients were selected from 2018 to 2020 . The inclusion criteria were patients who were intubated, an age $>18$ years, the presence of sICH on computerized tomographic (CT) scans, who had undergone spontaneous breathing trials with recorded breathing parameters. The exclusion criteria were a diagnosis of traumatic brain injury, vascular malformations on cerebral imaging, patients who had not undergone brain CT scans without contrast on admission, and patients whose families had only elected for comfort measures. The Institutional Review Board at the institution approved this study.

The initial patient data were recorded from the time of admission, including the Glasgow Coma Scale (GCS), intracerebral haemorrhage (ICH) score, and the National Institutes of Health (NIH) Stroke Scale. The cerebral CT scan evidence of intracerebral haemorrhages was characterized by location, laterality, volume, intraventricular haemorrhage, amount of midline shift, and hydrocephalus presence. O2 saturation, RSBI, NIF, partial pressure of oxygen $\left(\mathrm{pO}_{2}\right.$ ), partial pressure of carbon dioxide $\left(\mathrm{pCO}_{2}\right)$, bicarbonate $\left(\mathrm{HCO}_{3}\right)$, and

presence of cuff leak were recorded on each day of the spontaneous breathing trial. Patients were considered for extubation according to the criteria of RSBI <105, NIF <-25 cm H2O, and presence of cuff leak, along with a clinical judgment. Patient charts were then evaluated to determine which patients required reintubation and/or tracheostomy.

\section{Results}

Twenty-nine patients met the inclusion criteria and were evaluated in the study. The characteristics of their intracerebral haemorrhages are outlined in table 1 . As seen in table 2, of these 29 patients, 20 were extubated based on spontaneous breathing trial parameters and clinical judgment. The typical framework for parameters at our institution is the published standard of RSBI $<105$ and NIF $<-25 \mathrm{~cm} \mathrm{H}_{2} \mathrm{O}$ along with the presence of a cuff leak [7]; however, patients not meeting these requirements could undergo extubation if clinically indicated. Table 3 outlines the standard criteria' success rates at our hospital, along with various other cutoffs for both RSBI and NIF. As noted in the table, cuff leak and NIF <-30 $\mathrm{cm} \mathrm{H}_{2} \mathrm{O}$, independent of RSBI, led to $100 \%$ successful extubation rate. 


\section{Cureus}

\begin{tabular}{|c|c|}
\hline \multicolumn{2}{|l|}{ ICH Parameters } \\
\hline ICH Score & Patients $(n=29)$ \\
\hline 1 & 5 \\
\hline 2 & 14 \\
\hline 3 & 7 \\
\hline 4 & 3 \\
\hline 5 & 0 \\
\hline \multicolumn{2}{|l|}{ Laterality } \\
\hline Left & 20 \\
\hline Right & 8 \\
\hline Midline & 1 \\
\hline \multicolumn{2}{|l|}{ Location } \\
\hline Lobar & 5 \\
\hline Basal ganglia/thalamus & 23 \\
\hline Brainstem & 1 \\
\hline \multicolumn{2}{|l|}{ IVH } \\
\hline Yes & 20 \\
\hline No & 9 \\
\hline \multicolumn{2}{|l|}{ Hydrocephalus } \\
\hline Yes & 9 \\
\hline No & 20 \\
\hline Average volume & $20.5 \mathrm{ml}$ \\
\hline Average midline shift & $3.5 \mathrm{~mm}$ \\
\hline
\end{tabular}

TABLE 1: Characteristics of intracerebral haemorrhages.

$\mathrm{ICH}=$ intracerebral hemorrhage; $\mathrm{n}=$ number; $\mathrm{IVH}=$ intraventricular hemorrhage; $\mathrm{ml}=$ milliliters; $\mathrm{mm}=$ millimeters

Ventilation Status

Extubated

Successful

Reintubated

Tracheostomy
Patients ( $\mathrm{n}=29$ )

20

14

6

15

TABLE 2: Ventilation status of patient population. 


\section{Cureus}

\begin{tabular}{|c|c|c|c|}
\hline Parameter(s) & Total Patients & Successful Extubations & Percent success (\%) \\
\hline $\mathrm{RSBI}<105$ and $\mathrm{NIF}$ & 9 & 8 & 88.9 \\
\hline $\mathrm{RSBI}<85$ and $\mathrm{NIF}$ & 7 & 7 & 100.0 \\
\hline RSBI $<105$ & 17 & 12 & 70.6 \\
\hline $\mathrm{RSBl}<85$ & 14 & 10 & 71.4 \\
\hline NIF & 11 & 10 & 90.9 \\
\hline NIF & 9 & 9 & 100.0 \\
\hline
\end{tabular}

TABLE 3: Extubation parameters along with success rate expressed in percentage.

$\mathrm{RSBI}=$ rapid shallow breathing index; NIF = negative inspiratory force

Patients who failed extubation and ultimately underwent tracheostomy placement had various non-optimal spontaneous breathing parameters and continued to have non-optimal RSBI and NIF. Four patients met the criteria for extubation per cuff leak, RSBI and NIF, but ultimately were not extubated and underwent tracheostomy placement due to clinical judgment.

The positive predictive value for using NIF $<-30 \mathrm{~cm} \mathrm{H}_{2} 0$ is $100 \%$. The negative predictive value of using the latter is $50 \%$.

\section{Discussion}

The normal weaning criteria, RSBI $<105$ and NIF $<-25$ may not always apply to patients with intracerebral haemorrhages or other brain injuries $[8,11,12]$. Although a typical success rate of approximately $80 \%$ has been determined for extubation in patients who required prolonged mechanical ventilation [13], our patients were only successfully extubated $70 \%$ of the time. This further supports the notion that patients with a neurological injury who have an altered mental status, do not necessarily follow the normal paradigms seen in other ventilated patients. Additional care must be taken to prevent adverse outcomes from both too aggressive and not aggressive enough extubation. Patients risk developing tracheal and esophagal injuries from reintubation. Extended intubation can lead to pneumonia, more prolonged ICU stays, and the development of a poorer prognosis $[5,14]$.

The RSBI has been traditionally verified to have a positive predictive value (PPV) and negative predictive value (NPV) of $79 \%$ and $95 \%$, respectively, at a cutoff value of 105 when evaluating patients for extubation [7]. Prior studies have noted that neurosurgical patients are typically intubated for airway protection and not lung pathology and therefore, the RSBI is not necessarily a good predictor of extubation success. In the same study, only one patient that required reintubation had an RSBI $>105$, which would have predicted extubation success [15]. Due to the NIF being a measure of the inspiratory muscles, this measure may be the strongest indicator of extubation success $[7,16]$. The NIF considers a patient's innate ability to generate inspiratory force to create a breath along with their ability to have cognitive input to maximize their effort. As such, the NIF inherently measures a patient's ability to protect the airway than the RSBI better. As evidenced by our study, we can reach $100 \%$ success rate by altering the NIF goal independent of the RSBI criteria. Using NIF $<-30 \mathrm{~cm} \mathrm{H} 2 \mathrm{O}$ as criteria, we have a positive predictive value of $100 \%$ and a negative predictive value of $50 \%$, ensuring success. Even though the negative predictive value is low, patients do not suffer additional trauma and reintubation complications, which is more burdensome than temporary tracheostomy [6]. This further supports the notion that the NIF, in addition to cuff leak, maybe the most critical metric in determining successful extubation in these patients.

In patients that ultimately had a tracheostomy, the spontaneous breathing parameters fit within the newly proposed criteria, but other clinical factors determined the patients to not be candidates for extubation. These factors were the "standard" concerns for neurologically suppressed patients and included poor mentation as determined by the respiratory therapist's judgment. One notable incident leading to reintubation in the presence of a new or worsening bleed, causing a deterioration in neurological status. These patients may again become unable to protect their airway and require reintubation; the RSBI and NIF cannot account for the risk of this occurring. This highlights the importance of clinical judgment when deciding to extubate. Clinical judgment typically supersedes numerical evaluation through spontaneous breathing trials and can lead to patients not being extubated at a high enough rate [12]. It is essential to note the subjectivity in clinical judgment, and having criteria with a high predictive value can help mitigate the concerns of operator differences. 
Our study is not without its limitations. The patient population can be limited due to the nature in which patients arrive at the county hospital. Many patients have private insurance, requiring transfer to in-network facilities once stabilized, and many patients were transferred before extubation. A larger number of patients could provide further support for our conclusions. Additionally, many patients did not have reliable past medical histories and prior lung pathologies, which could have contributed to extubation success and failure due to an overabundance of caution about clinical judgment [13]. Analyzing these variables and others such as the route of intubation and degree of sedation in the failed extubations would help evaluate more possible causes instead of only assessing spontaneous breathing trial parameters [14].

\section{Conclusions}

Extubation success for all critical care patients is of vital importance. The traditional weaning parameters of RSBI and NIF have been valuable in predicting this success; however, neurosurgical patients do not necessarily follow the same patterns of extubation criteria. We have demonstrated that RSBI may not be as vital in decision making as the use of NIF $<-30 \mathrm{~cm} \mathrm{H}_{2} \mathrm{O}$. A stricter goal of a NIF $<-30 \mathrm{~cm} \mathrm{H}_{2} \mathrm{O}$ leads to a far higher success rate for extubations. Further prospective studies are needed to continue to test the weaning criteria in extubating neurosurgical patients with sICH.

\section{Additional Information \\ Disclosures}

Human subjects: Consent was obtained by all participants in this study. Arrowhead Regional Medical Center issued approval n/a. IRB Exempt. Animal subjects: All authors have confirmed that this study did not involve animal subjects or tissue. Conflicts of interest: In compliance with the ICMJE uniform disclosure form, all authors declare the following: Payment/services info: All authors have declared that no financial support was received from any organization for the submitted work. Financial relationships: All authors have declared that they have no financial relationships at present or within the previous three years with any organizations that might have an interest in the submitted work. Other relationships: All authors have declared that there are no other relationships or activities that could appear to have influenced the submitted work.

\section{References}

1. Szeder V, Ortega-Gutierrez S, Ziai W, Torbey MT: The TRACH score: clinical and radiological predictors of tracheostomy in supratentorial spontaneous intracerebral hemorrhage. Neurocrit Care. 2010, 13:40-46. 10.1007/s12028-010-9346-1

2. Burtin P, Bollaert PE, Feldmann L, Nace L, Lelarge P, Bauer P, Larcan A: Prognosis of stroke patients undergoing mechanical ventilation. Intensive Care Med. 1994, 20:32-36. 10.1007/BF02425052

3. Gujjar AR, Deibert E, Manno EM, Duff S, Diringer MN: Mechanical ventilation for ischemic stroke and intracerebral hemorrhage: indications, timing, and outcome. Neurology. 1998, 51:447-451. 10.1212/wnl.51.2.447

4. Koh WY, Lew TWK, Chin NM, Wong MFM: Tracheostomy in a neuro-intensive care setting: indications and timing. Anaesth Intensive Care. 1997, 25:365-368. 10.1177/0310057X9702500407

5. Coplin WM, Pierson DJ, Cooley KD, Newell DW, Rubenfeld GD: Implications of extubation delay in braininjured patients meeting standard weaning criteria. Am J Respir Crit Care Med. 2000, 161:1530-1536. 10.1164/ajrccm.161.5.9905102

6. Karaniia N, Nordquist D, Stevens R, Nyquist P: A clinical description of extubation failure in patients with primary brain injury. Neurocrit Care. 2011, 15:4-12. 10.1007/s12028-011-9528-5

7. Aziz MM, Haroun MA, Meligy B, Saad MN: Comparison between RSBI (Rapid Shallow Breathing Index) and NIF (Negative Inspiratory Force) as predictors of extubation failure in mechanically ventilated pediatric patients. RJMMS. 2019, 14:10-20.

8. Ko R, Ramos L, Chalela JA: Conventional weaning parameters do not predict extubation failure in neurocritical care patients. Neurocrit Care. 2009, 10:269-273. 10.1007/s12028-008-9181-9

9. dos Reis HFC, Almeida MLO, da Silva MF, Moreira JO, de S. Rocha M: Association between the rapid shallow breathing index and extubation success in patients with traumatic brain injury. Rev Bras Ter Intensiva. 2013, 25:212-217. 10.5935/0103-507X.20130037

10. Vidotto MC, Sogame LCM, Calciolari CC, Nascimento OA, Jardim JR: The prediction of extubation success of postoperative neurosurgical patients using frequency-tidal volume ratios. Neurocrit Care. 2008, 9:83-89. 10.1007/s12028-008-9059-X

11. Huttner HB, Kohrmann M, Berger C, Georgiadis D, Schwab S: Predictive factors for tracheostomy in neurocritical care patients with spontaneous supratentorial hemorrhage. Cerebrovasc Dis. 2006, 21:159-165. $10.1159 / 000090527$

12. Namen AM, Ely EW, Tatter SB, et al.: Predictors of successful extubation in neurosurgical patients . Am J Respir Crit Care Med. 2001, 163:658-664. 10.1164/ajrccm.163.3.2003060

13. Morganroth ML, Grum CM: Weaning from Mechanical Ventilation. J Intensive Care Med. 1988, 3:109-120. $10.1177 / 088506668800300207$

14. Chevron V, Ménard JF, Richard JC, Girault C, Leroy J, Bonmarchand G: Unplanned extubation: risk factors of development and predictive criteria for reintubation. Crit Care Med. 1998, 26:1049-1053. 10.1097/00003246-199806000-00026

15. Karthika M, Al Enezi FA, Pillai LV, Arabi YM: Rapid shallow breathing index. Ann Thorac Med. 2016, 11:167176. $10.4103 / 1817-1737.176876$ 


\section{Cureus}

16. Meade M, Guyatt G, Cook D, et al.: Predicting success in weaning from mechanical ventilation . Chest. 2001, 120:400S-24S. 10.1378/chest.120.6_suppl.400S 